\title{
Virus-Information Coevolution Spreading Dynamics on Multiplex Networks
}

\author{
Jian Wang $\mathbb{D},{ }^{1,2,3}$ Xiaolin Qin, ${ }^{2}$ and Hongying Fang $\mathbb{D}^{4}$ \\ ${ }^{1}$ School of Computer Science and Technology, University of Chinese Academy of Sciences, Beijing 100049, China \\ ${ }^{2}$ Chengdu Institute of Computer Applications, Chinese Academy of Sciences, Chengdu 610041, China \\ ${ }^{3}$ College of Computer Science and Technology, Chongqing University of Posts and Telecommunications, Chongqing 400065, China \\ ${ }^{4}$ College of Mathematics and Statistics, Chongqing Jiaotong University, Chongqing 400074, China
}

Correspondence should be addressed to Hongying Fang; 284199018@qq.com

Received 10 December 2020; Revised 22 December 2020; Accepted 10 February 2021; Published 5 March 2021

Academic Editor: Ning Cai

Copyright (C) 2021 Jian Wang et al. This is an open access article distributed under the Creative Commons Attribution License, which permits unrestricted use, distribution, and reproduction in any medium, provided the original work is properly cited.

\begin{abstract}
Virus and information spreading dynamics widely exist in complex systems. However, systematic study still lacks for the interacting spreading dynamics between the two types of dynamics. This paper proposes a mathematical model on multiplex networks, which considers the heterogeneous susceptibility and infectivity in two subnetworks. By using a heterogeneous meanfield theory, we studied the dynamic process and outbreak threshold of the system. Through extensive numerical simulations on artificial networks, we find that the virus's spreading dynamics can be suppressed by increasing the information spreading probability, decreasing the protection power, or decreasing the susceptibility and infectivity.
\end{abstract}

\section{Introduction}

Coevolution spreading dynamics, ranging from cyberspace security to epidemic contagions, widely exist in the natural systems, in which there are at least two spreading dynamics evolving and interacting simultaneously $[1,2]$. For instance, the virus's information is always spreading on the social network when a computer virus spreads on the Internet. The users whose computers are not infected by the virus will install antivirus software and patches to protect their computers from being infected by the virus [3-6]. In this way, computer viruses can be prevented from spreading widely. Another example is the spreading of the epidemic in society. When a global pandemic was spreading, various kinds of information about the pandemic, such as protecting healthy individuals from infection, spreading on social networks will suppress the pandemic [7-9].

Researchers from the field of computer science and network science have developed some successful mathematical models to model such coevolution spreading dynamics. The state of the art in this field is reviewed in a recent paper by Wang et al. [1]. Historically, Newman [10] studied two viruses spreading on the same computer network in succession, where the two viruses follow the susceptibleinfected-recovered model, and the second virus can only infect the remaining susceptible nodes. Using a bond percolation theory, he revealed that a global outbreak of the second virus is possible only if the susceptible nodes form a large cluster of connections and the outbreak threshold of the second virus is much higher than the threshold of the first. Newman and Ferrario [11] further discussed a different situation, i.e., the second virus can only spread on those infected nodes by the first virus. They found that the second virus's outbreak size can be suppressed by decreasing the spreading probability of the first virus. In reality, the spreading dynamics are always simultaneous. Karrer and Newman [12] proposed a model to include this factor and studied the phase transition by using a competing percolation theory.

The two spreading dynamics always evolve on different networks; that is to say, we should use multiplex or multilayer networks to describe the network topology of the coevolution spreading [13-18]. Previous studies have revealed that the topology of multilayer networks markedly affect the dynamics, 
such as cascading failures [19-21], virus spreading [22, 23], controllability [24], and synchronization [25-27]. For virusinformation spreading on multiplex networks, Granell and her colleagues [8] used an unaware-aware-unaware-susceptibleinfected-susceptible (UAU-SIS) model. They revealed a metacritical critical point, above which the global virus will break out by using a generalized Markovian approach. Based on the research framework in Reference [8], researchers studied the global information [8], network topology [28, 29], and different interacting mechanisms $[30,31]$ on the virus spreading. Wang et al. revealed the asymmetric interaction between the virus and information spreading dynamics. They used a susceptible-informed-recovered-susceptible-infected-recovered-vaccination (SIR-SIRV) model. They found that the information can suppress the virus spreading greatly, especially when there is a positive correlation between layers.

Many real-world data analyses proved that the spreading dynamics on the network are heterogeneous. There are three aspects. On the one hand, the network topology is heterogeneous, e.g., heterogeneous degree distribution. Scholars revealed that heterogeneous degree distribution could decrease the virus's outbreak threshold spreading [32-34]. An important result is that Pastor-Satorras and Vespignani [32] used a heterogeneous mean-field theory to describe the computer virus spreading on the Internet and revealed that the existence of some hubs may make the outbreak threshold vanish. On the other hand, infectivity and susceptibility are heterogeneous since different computers have distinct circumstances. Miller [35] revealed that the global virus is more likely to break out for homogeneous infectivity when the average transmissibility is fixed. In addition, he found that the attack rate was highest when the susceptibility was homogeneous and lowest when the variance was maximum. Lastly, the virus and information always transmit through different networks. Generally, the virus spreads on the computer network and the information transmits on the social network. Therefore, the virus-information dynamics spreading on two-layered multiplex networks are more realistic. Previous paragraphs have stated the state-of-the-art progresses for virus-information spreading on multiplex networks. To our best knowledge, systematic study still lacks for the interacting spreading dynamics including the above three aspects. In the paper, we first describe the mathematical model in Section 2. In Section 3, we develop a heterogeneous mean-field theory to describe the spreading dynamics. In Section 4, we perform extensive numerical simulations. Finally, we conclude the paper in Section 5.

\section{Model Descriptions}

In this section, we propose the virus-information coevolution spreading model on computer-social network $M$. We first introduce the computer-social network and then present the virus-information spreading model.

2.1. Computer-Social Network. We denote the two subnetworks as $L_{1}$ and $L_{2}$, respectively. The computer virus spreads on subnetwork $L_{1}$, and the information spreads on subnetwork $L_{2}$. In subnetwork $L_{1}\left(L_{2}\right)$, nodes represent the computers (users), and the edges stand for the relationships among computers (users). To build the two-layered complex networks, we use the following steps: (i) assigning the subnetwork sizes $N_{1}=N_{2}=N$; (ii) building subnetworks $L_{1}$ and $L_{2}$ by using the uncorrelated configuration model [36] (the degree distributions of subnetworks $L_{1}$ and $L_{2}$ are $P_{1}\left(k_{1}\right)$ and $P_{2}\left(k_{2}\right)$, respectively); and (iii) randomly matching nodes in two subnetworks. Specifically, we build an interlayer connection $e$ for node $i_{1}$ and $i_{2}$, which means that the user $i_{2}$ uses computer $i_{1}$. By using the above methods, there are node inter- and intradegree correlations. As shown in Figure 1, we illustrate the computer-social network.

Mathematically, the computer-social network $M$ can be represented by a adjacency matrix $U=\left(\begin{array}{cc}A^{1} & A^{12} \\ A^{21} & A^{2}\end{array}\right)$, where $A^{1}$ and $A^{2}$, respectively, stand for the adjacency matrixes of subnetworks $L_{1}$ and $L_{2}$. An element $A_{i j}^{x}=1$ of subnetwork $x \in\{1,2\}$ means that nodes $i_{x}$ and $j_{x}$ are connected. The matrixes $A^{12}$ and $A^{21}$ are the adjacency matrixes of interlayer network, where $A_{i_{1} i_{2}}^{12}=1$ means that node $i_{1}$ uses computer $i_{2}$. Note that $A_{i_{1} i_{2}}^{12}=A_{i_{2} i_{1}}^{21}$ for any values of $i_{1}$ and $i_{2}$. The average degrees of the two subnetworks can be denoted as $\left\langle k_{1}\right\rangle=$ $\sum_{i, j} A_{i j}^{1}=\sum_{k_{1}} k_{1} P_{1}\left(k_{1}\right)$ and $\left\langle k_{2}\right\rangle=\sum_{i, j} A_{i j}^{2}=\sum_{k_{2}}{ }_{2}{ }_{2} P_{2}\left(k_{2}\right)$, respectively.

2.2. Virus-Information Spreading Model. We here adopt a susceptible-infected-recovered (SIR) model to describe the virus spreading on subnetwork $L_{1}$. A node in the susceptible state means that it does not get infected by the computer virus. An infected node represents that it is infected by the virus and can transmit it to one of its neighbors. A node in the recovered state means that it has recovered and does not change its state.

For the information spread on subnetwork $L_{2}$, we consider using the irreversible susceptible-informed-recovered (SIR) model [37]. A susceptible node means that it does not obtain information about the virus. An informed node indicates that it knows information about the virus and is willing to share it with its neighbors. A node in the recovered state means that it loses interest in the information and will not transmit it to its neighbors. In this paper, we denote the virus-information coevolution spreading as a SIR-SIR model.

The virus-information coevolution spreading dynamic evolves as follows. Initially, we randomly select a node $i_{1}$ in subnetwork $L_{1}$, that is to say, the computer virus infects node $i_{1}$. The corresponding node $i_{2}$, i.e., the user of computer $i_{1}$, is also set to be the infected state, since the user can release the information of his infection to his neighbors. At every time step $t$, each infected node $v_{1}$ in subnetwork $L_{1}$ tries to transmit the computer virus to one of its neighbors $u_{1}$, since every infected node usually communicates with one neighbor at a short time interval. In reality, the infection transmission depends on the "source" and "target" nodes [35]. That is to say, the infectivity and susceptibility of the system are distinct for different nodes. To include this factor, 


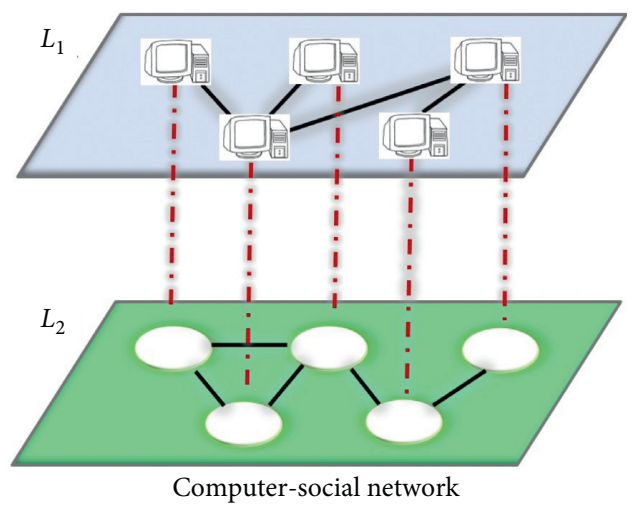

FIGURE 1: Illustration of computer-social networks. Subnetwork $L_{1}$ represents the computer network, and subnetwork $L_{2}$ stands for the social network.

we assume that nodes' infectivity and susceptibility depend on the degree of nodes. More specifically, the infectivity of node $v_{1}$ with degree $k_{1}$ is

$$
\omega_{k_{1}}^{1}=1-\left(1-\alpha_{1}\right)^{k_{1}},
$$

where $\alpha_{1}$ is the unit infectivity for a node with degree 1 . Similarly, the susceptibility of node $u_{1}$ with degree $k_{1}^{\prime}$ is

$$
\varpi_{k_{1}^{\prime}}^{1}=1-\left(1-\alpha_{1}^{\prime}\right)^{k_{1 \prime}},
$$

where $\alpha_{1}^{\prime}$ is the unit susceptibility for node with degree 1 . Varying the values of $\alpha_{1}$ and $\alpha_{1}^{\prime}$, we get different infectivities and susceptibilities of the system. If node $u_{1}$ is susceptible, two different situations should be considered. (i) If the user $u_{2}$ of computer $u_{1}$ is in the susceptible state, the computer $u_{1}$ infects the virus with probability $\lambda_{1}$. Meanwhile, node $u_{2}$ obtains the information. Otherwise, $u_{2}$ is in the infected or recovered state, and nothing happens. (ii) If the user $u_{2}$ has already obtained the information before, the computer $u_{1}$ is infected by the virus with probability $q \lambda_{1}$, where $0 \leq q \leq 1$. We here use the parameter $q$ to describe the degree of protection when a user knows the virus's spreading. The smaller the value of $q$, the stronger the protection against computer viruses. Every infected node recovers with probability $\gamma_{1}$.

The information about the virus spread on the subnetwork $L_{2}$ is as follows. At each time step $t$, every informed node $v_{2}$ transmits the information to one of its neighbor $u_{2}$ in subnetwork $L_{2}$ depending on the infectivity of $v_{2}$ and the susceptibility of $u_{2}$. The infectivity of $v_{2}$ with degree $k_{2}$ is

$$
\omega_{k_{2}}^{2}=1-\left(1-\alpha_{2}\right)^{k_{2}},
$$

where $\alpha_{2}$ is the unit infectivity. The susceptibility of $u_{2}$ with degree $k_{2}^{\prime}$ is

$$
\varpi_{k_{2}^{\prime}}^{2}=1-\left(1-\alpha_{2}^{\prime}\right)^{k_{2 \prime}},
$$

where $\alpha_{2}^{\prime}$ is the unit susceptibility. The infection probability is $\lambda_{2}$. Finally, every informed node loses interest in transmitting the information with probability $\gamma_{2}$. The spreading ends when there are no nodes in the infected or informed state. In Table 1, we present the definitions of parameters and abbreviations.

\section{Heterogeneous Mean-Field Theory}

In this section, we develop a heterogeneous mean-field approach to describe the evolution of the virus-information spreading dynamics. In theory, we assume that nodes with same degrees have the same infection probability in statistical $[32,33,38,39]$. In other words, the probability of nodes with the degree $k$ is the same as each other.

We use the following parameters to describe the coevolution process. Denote $s_{k_{1}}^{1}(t), \rho_{k_{1}}^{1}(t)$, and $r_{k_{1}}^{1}(t)$ as the probability that a node with degree $k_{1}$ is in the susceptible, infected, and recovered states at time $t$ in subnetwork $L_{1}$, respectively. Similarly, we denote $s_{k_{2}}^{2}(t), \rho_{k_{2}}^{2}(t)$, and $r_{k_{2}}^{2}(t)$ as the probability of node with degree $k_{2}$ in the susceptible, informed, and recovered states at time $t$ in subnetwork $L_{2}$, respectively. Considering the degree distributions of subnetworks $L_{1}$ and $L_{2}$, we know the fraction of nodes in each state. For instance, the fraction of nodes in the susceptible state at time $t$ is $S_{1}(t)=\sum_{k_{1}} P_{1}\left(k_{1}\right) s_{k_{1}}^{1}(t)$. In the final state, i.e., $t \longrightarrow \infty$, the fraction of nodes in the susceptible state is $S_{1}(\infty) \equiv S_{1}$.

Now we derive the expressions of the probability of nodes in each state. We know that $s_{k_{1}}^{1}(t)$ decreases with time $t$ when nodes are infected by the virus. A susceptible computer $u_{1}$ with degree $k_{1}$ infected by the virus has two situations. (1) The corresponding node (i.e., the user) $u_{2}$ of node $u_{1}$ is in the susceptible state. In this situation, the infection transmitted to node $u_{1}$ should fulfill two necessary conditions.

(i) An infected neighbor $v_{1}$ of node $u_{1}$ contacts node $u_{1}$. In uncorrelated complex networks, the probability of node $u_{1}$ connecting to an infected neighbor $v_{1}$ with degree is $k_{1}^{\prime}$ is $\left(\left(k_{1}^{\prime}-1\right) / \sum_{k_{1}} k_{1} P_{1}\left(k_{1}\right)\right)$, where $k_{1}^{\prime}$ is the degree of node $v_{1}$. Considering the degree distribution of subnetwork $L_{1}$, the average probability that a node connects to an infected neighbor through an edge is

$$
\Theta_{1}(t)=\frac{1}{\left\langle k_{1}\right\rangle} \sum_{k_{1}^{\prime}} \omega_{k_{1}^{\prime}}^{1}\left(k_{1}^{\prime}-1\right) P_{1}\left(k_{1}^{\prime}\right) \rho_{k_{1}^{\prime}}^{1}(t) .
$$

(ii) The infection is transmitted successfully with probability $\lambda_{1}$. According to the description of the model, for a node $u_{1}$ in the susceptible state, its corresponding node must also be in the susceptible state. However, the opposite situation does not always exist. Combining conditions (i) and (ii), we know situation (1) happens with probability $\lambda_{1} k_{1} \bigoplus_{k_{1}}^{1} s_{k_{1}}^{1}(t) \Theta_{1}(t)$. Situation (2) indicates that node $u_{2}$ of node $u_{1}$ is in the informed state. Using a similar discussion with situation (1), we obtain the probability of situation (2) as follows: $q \lambda_{1} k_{1} \Phi_{k_{1}}^{1} s_{k_{1}}^{1}(t) \Theta_{1}(t)\left[\sum_{k_{2}} P_{2}\left(k_{2}\right) \lambda_{2} k_{2} \Phi_{k_{2}}^{2} \Theta_{2}(t)\right]$, where $\sum_{k_{2}} P_{2}\left(k_{2}\right) \lambda_{2} k_{2} \omega_{k_{2}}^{2} \Theta_{2}(t)$ is the probability that the corresponding node $u_{2}$ of $u_{1}$ is informed by neighbors in subnetwork $L_{2}$ at time $t . \Theta_{2}(t)$ will be defined later. The rate equation of $s_{k_{1}}^{1}(t)$ is 
TABLE 1: Definitions of parameters and abbreviations.

\begin{tabular}{|c|c|}
\hline Parameter & Definition \\
\hline$N_{1}$ & Subnetwork size of computer network $L_{1}$ \\
\hline$N_{2}$ & Subnetwork size of social network $L_{2}$ \\
\hline$P_{1}^{2}\left(k_{1}\right)$ & Degree distribution of computer network $L_{1}$ \\
\hline$P_{2}\left(k_{2}\right)$ & Degree distribution of social network $L_{2}$ \\
\hline$\left\langle k_{1}\right\rangle$ & Average degree of computer network $L_{1}^{2}$ \\
\hline$\left\langle k_{2}\right\rangle$ & Average degree of social network $L_{2}$ \\
\hline$\omega_{k_{1}}^{1}$ & Infectivity of node $v_{1}$ with degree $k_{1}$ in subnetwork $L_{1}$ \\
\hline$\omega_{k_{2}}^{2}$ & Infectivity of node $v_{2}$ with degree $k_{2}$ in subnetwork $L_{2}$ \\
\hline$\varpi_{k_{1}^{\prime}}^{n_{2}^{2}}$ & Susceptibility of $u_{1}$ with degree $k_{1}^{\prime}$ in subnetwork $L_{1}$ \\
\hline$\omega_{k_{2}^{\prime}}^{k_{1}}$ & Susceptibility of $u_{2}$ with degree $k_{2}^{\prime}$ in subnetwork $L_{2}$ \\
\hline$\alpha_{1}$ & Unit infectivity in subnetwork $L_{1}$ \\
\hline$\alpha_{2}$ & Unit infectivity in subnetwork $L_{2}$ \\
\hline$\alpha_{1}^{\prime}$ & Unit susceptibility in subnetwork $L_{1}$ \\
\hline$\alpha_{2}^{\prime}$ & Unit susceptibility in subnetwork $L_{2}$ \\
\hline$q$ & Protection power \\
\hline$\lambda_{1}$ & Computer virus transmission probability \\
\hline$\lambda_{2}$ & Information transmission probability \\
\hline$\gamma_{1}$ & Computer virus recovery probability \\
\hline$\gamma_{2}$ & Information recovery probability \\
\hline$s_{k_{1}}^{1}(t)$ & Probability of node with degree $k_{1}$ in the susceptible state at time $t$ in subnetwork $L_{1}$ \\
\hline$\rho_{k_{1}}^{1}(t)$ & Probability of node with degree $k_{1}$ in the infected state at time $t$ in subnetwork $L_{1}$ \\
\hline$r_{k_{1}}^{1}(t)$ & Probability of node with degree $k_{1}$ in the recovered state at time $t$ in subnetwork $L_{1}$ \\
\hline$s_{k}^{2}(t)$ & Probability of node with degree $k_{2}$ in the susceptible state at time $t$ in subnetwork $L_{2}$ \\
\hline$\rho_{k_{2}}^{2}(t)$ & Probability of node with degree $k_{2}$ in the infected state at time $t$ in subnetwork $L_{2}$ \\
\hline$r_{k_{2}}^{2}(t)$ & Probability of node with degree $k_{2}$ in the recovered state at time $t$ in subnetwork $L_{2}$ \\
\hline$\Theta_{1}^{2}(t)$ & Average probability that a node connects to an infected neighbor through an edge in subnetwork $L_{1}$ \\
\hline$\Theta_{2}(t)$ & Average probability that a node connects to an informed neighbor through an edge in subnetwork $L_{2}$ \\
\hline
\end{tabular}

$$
\frac{\mathrm{d} s_{k_{1}}^{1}(t)}{\mathrm{d} t}=-\lambda_{1} k_{1} \oplus_{k_{1}}^{1} \Theta_{1}(t)\left[s_{k_{1}}^{1}(t)+q \sum_{k_{2}} P_{2}\left(k_{2}\right) \lambda_{2} k_{2} \Theta_{k_{2}}^{2} \Theta_{2}(t)\right] .
$$

$$
\begin{aligned}
& \text { The evolution of } \rho_{k_{1}}^{1}(t) \text { is } \\
& \begin{aligned}
\frac{\mathrm{d} \rho_{k_{1}}^{1}(t)}{\mathrm{d} t}= & \lambda_{1} k_{1} \oplus_{k_{1}}^{1} \Theta_{1}(t)\left[s_{k_{1}}^{1}(t)+q \sum_{k_{2}} P_{2}\left(k_{2}\right) \lambda_{2} k_{2} \Theta_{k_{2}}^{2} \Theta_{2}(t)\right] \\
& -\gamma_{1} \rho_{k_{1}}^{1}(t),
\end{aligned}
\end{aligned}
$$

where $\gamma_{1} \rho_{k_{1}}^{1}(t)$ is the fraction of nodes recovered at time $t$. Finally, the evolution of $r_{k_{1}}^{1}(t)$ is

$$
\frac{\mathrm{d} r_{k_{1}}^{1}(t)}{\mathrm{d} t}=\gamma_{1} \rho_{k_{1}}^{1}(t)
$$

According to equations (6)-(8), we obtain the evolution of computer virus spreading on subnetwork $L_{1}$.

Now, we study the rate equations of the information about the virus spreading on social network $L_{2}$. There are two different situations for the reduction of $s_{k_{2}}^{2}(t)$. For the first situation, the susceptible node $u_{2}$ with degree $k_{2}$ is infected by its informed neighbor $v_{2}$. The infection probability is $\lambda_{2} k_{2} \omega_{k_{2}}^{2} s_{k_{2}}^{2}(t) \Theta_{2}(t)$, where $\Theta_{2}(t)$ denotes the probability of an edge connecting to an informed neighbor in subnetwork $L_{2}$. The expression of $\Theta_{2}(t)$ can be expressed as

$$
\Theta_{2}(t)=\frac{1}{\left\langle k_{2}\right\rangle} \sum_{k_{2}^{\prime}} \omega_{k_{2}^{\prime}}^{2}\left(k_{2}^{\prime}-1\right) P_{2}\left(k_{2}^{\prime}\right) \rho_{k_{2}^{\prime}}^{2}(t)
$$

The second situation of node $u_{2}$ obtaining the information is that the corresponding node $u_{1}$ of $u_{2}$ is infected by the computer virus through an edge of $u_{1}$ with probability $\lambda_{1} k_{1} \omega_{k_{1}}^{1} s_{k_{2}}^{2}(t) \Theta_{1}(t)$. Since $u_{2}$ and $u_{1}$ are randomly coupled, the averaged infection probability of $u_{1}$ is $\lambda_{1}\left\langle k_{1}\right\rangle\left\langle\Phi_{k_{1}}^{1}\right\rangle s_{k_{2}}^{2}(t) \Theta_{1}(t)$, where $\left\langle\Phi_{k_{1}}^{1}\right\rangle=\sum_{k_{1}} P_{1}\left(k_{1}\right) \varpi_{k_{1}}^{1}$. Combining the two situations, we obtain the rate equation of $s^{2} k_{2}(t)$ as

$$
\frac{\mathrm{d} s_{k_{2}}^{2}(t)}{\mathrm{d} t}=-s_{k_{2}}^{2}(t)\left[\lambda_{2} k_{2} \Theta_{k_{2}}^{2} \Theta_{2}(t)+\lambda_{1}\left\langle k_{1}\right\rangle\left\langle\Theta_{k_{1}}^{1}\right\rangle \Theta_{1}(t)\right] .
$$

With the similar discussion about the virus spreading on subnetwork $L_{1}$, we have 


$$
\frac{\mathrm{d} \rho_{k_{2}}^{2}(t)}{\mathrm{d} t}=s_{k_{2}}^{2}(t)\left[\lambda_{2} k_{2} \Theta_{k_{2}}^{2} \Theta_{2}(t)+\lambda_{1}\left\langle k_{1}\right\rangle\left\langle\Theta_{k_{1}}^{1}\right\rangle \Theta_{1}(t)\right]-\gamma_{2} \rho_{k_{2}}^{1}(t),
$$

$\frac{\mathrm{d} r_{k_{2}}^{2}(t)}{\mathrm{d} t}=\gamma_{2} \rho_{k_{2}}^{2}(t)$

With the above equations, we know the fraction of nodes in each state at subnetworks $L_{1}$ and $L_{2}$.

In the following, we study the global outbreak conditions of the computer virus and information spreading. For global information outbreak condition, we can linearize equations (7) and (11) around the initial conditions, i.e., $s_{k_{1}}^{1} \approx 1$, $s_{k_{2}}^{2} \approx 1$. We know that $s_{k_{1}}^{1}=1, s_{k_{2}}^{2}=1, \rho_{k_{1}}^{1}=0$, and $\rho_{k_{2}}^{2}=0$ are trivial solutions. Denote a vector $\vec{\rho}=\left(\overrightarrow{\rho_{1}}, \overrightarrow{\rho_{2}}\right)^{T}$, where $\overrightarrow{\rho_{1}}=\left(\rho_{k_{1}=1}^{1}, \ldots, \rho_{k_{1, \max }}^{1}\right), \overrightarrow{\rho_{2}}=\rho_{k_{2}=1}^{2}, \ldots, \rho_{k_{2, \max }}^{2}$, and $k_{1, \max }^{1}$ and $k_{2 \text {, max }}^{2}$ represent the maximal degrees of subnetworks $L_{1}$ and $L_{2}$, respectively. We perform a Taylor expansion for equations (7) and (11) at $s_{k_{1}}^{1}=1, s_{k_{2}}^{2}=1, \rho_{k_{1}}^{1}=0$, and $\rho_{k_{2}}^{2}=0$ and neglect the high order of $\vec{\rho}$. We have

$$
\frac{\mathrm{d} \vec{\rho}}{\mathrm{d} t}=\mathbf{J} \vec{\rho}
$$

where $\mathbf{J}$ is the Jacobian matrix. The expression of $\mathbf{J}$ is

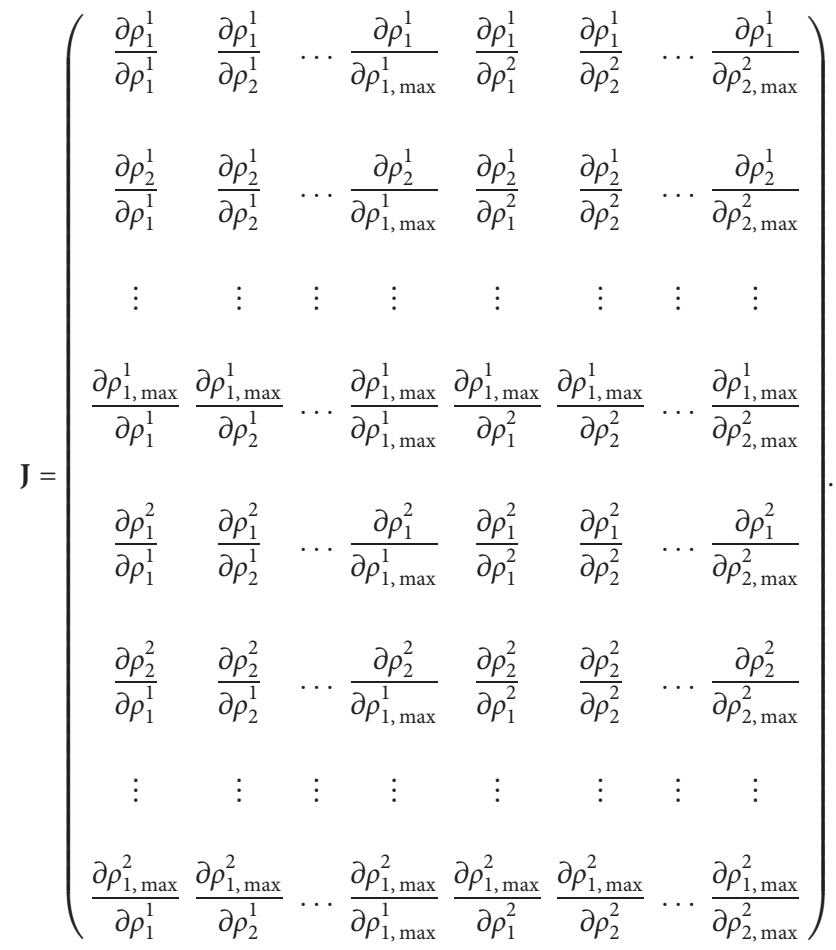

The Jacobian matrix $\mathbf{J}$ can be further expressed as block matrix as

$$
\mathbf{J}=\left(\begin{array}{ll}
C^{1} & D^{2} \\
D^{1} & C^{2}
\end{array}\right)
$$

where dimensions of $C^{1}, C^{2}, D^{1}$, and $D^{2}$ are $k_{1, \max } \times k_{1, \max }$, $k_{2, \text { max }} \times k_{2, \text { max }}, k_{2, \text { max }} \times k_{1, \text { max }}$, and $k_{1, \text { max }} \times k_{2, \text { max }}$, respectively. When the global information on subnetwork $L_{2}$ breaks out, the largest eigenvalue of $\mathbf{J}$ is larger than zero. The global information outbreak condition is

$$
\Lambda_{1}(\mathbf{J})=0 \text {, }
$$

where $\Lambda_{1}(\mathbf{J})$ is the largest eigenvalue of $\mathbf{J}$. For the virus outbreak condition, we cannot solve it directly. When the network is extensive, we can use the competing percolation theory [40]. That is to say, we can process the information spreading on subnetwork $L_{2}$ first and then the virus spreading on subnetwork $L_{1}$.

\section{Simulation Results}

In this section, we perform numerical simulations to study the virus-information spreading dynamics on computer-social network. To build the computer-social network, we use the uncorrelated configuration model [36]. We set the degree distributions of subnetworks $L_{1}$ and $L_{2}$ as $P_{1}\left(k_{1}\right) \sim k^{-} \chi_{1}$ and $P_{2}\left(k_{2}\right) \sim k^{-} \chi_{2}$, respectively, where $\chi_{1}$ and $\chi_{2}$, respectively, represent the degree exponents. There is no inter- and intralayer degree-degree correlations. In numerical simulations, we set the average degrees of the two subnetworks as $\left\langle k_{1}\right\rangle=\left\langle k_{2}\right\rangle=8$, the network sizes as $N_{1}=N_{2}=10^{4}$, and the degree exponent as $\chi_{1}=\chi_{2}=3.0$. We set $\alpha_{1}=\alpha_{1}^{\prime}$ and $\alpha_{2}=\alpha_{2}^{\prime}$. Initially, we randomly select 5 seeds in subnetwork $L_{1}$. All results presented in this paper are averaged over at least 2000 times.

We first study the virus and information spreading sizes, respectively, denoted as $R_{1}=1-S_{1}$ and $R_{2}=1-S_{2}$, versus virus transmission probability $\lambda_{1}$ as shown in Figure 2 . We find that $R_{1}$ increases with $\alpha_{1}=\alpha_{2}$, i.e., the virus is more likely to spread when the infectivity and susceptibility are large. Specifically, we note that the virus cannot spread for any values of $\lambda_{1}$ when $\alpha_{1}=\alpha_{2}$ are small, e.g., $\alpha_{1}=\alpha_{2}=0.0$ and 0.2. When $\alpha_{1}=$ $\alpha_{2}$ are large enough, enlarging their values cannot promote the virus spreading. When comparing the effects of information spreading on virus spreading, i.e., increasing $\lambda_{2}$, the virus spreading can be suppressed, as shown in Figures 2(a)-2(d). That is to say, to contain the virus spreading, we can transmit more information about the virus on the social network. For the information spreading on subnetwork $L_{2}$, i.e., the social network, we find that $R_{2}$ increases with $\lambda_{1}, \lambda_{2}$, and $\alpha_{1}=\alpha_{2}$, since the users have more chances to obtain the information.

In Figure 3, we further investigate the effects of protection power $q$ on the virus-information spreading for different values of $\alpha_{1}=\alpha_{2}$ and $\lambda_{2}$. Generally speaking, we find similar results with that discussed in Figure 2. When the protection power is large, we find that the virus spreading size is relatively smaller, i.e., $R_{1}(q=0.8) \geq R_{1}(q=0.5)$, since the susceptible nodes are less likely to be infected by neighbors. We also note that $R_{2}(q=$ 


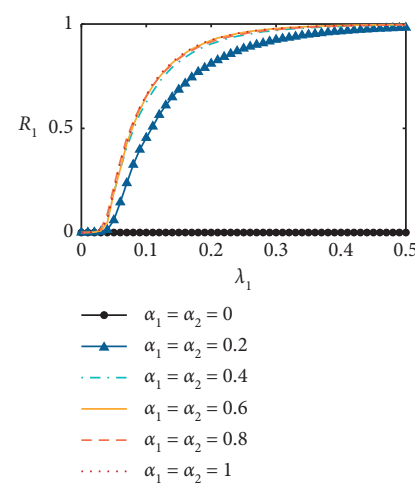

(a)

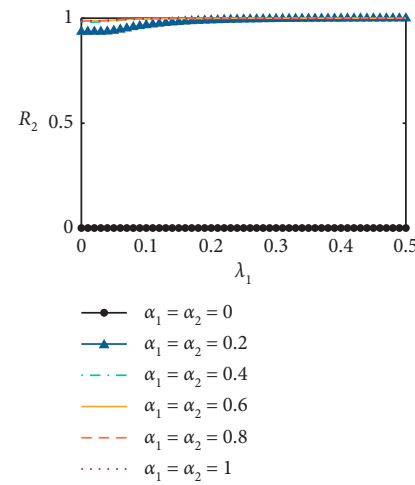

(e)
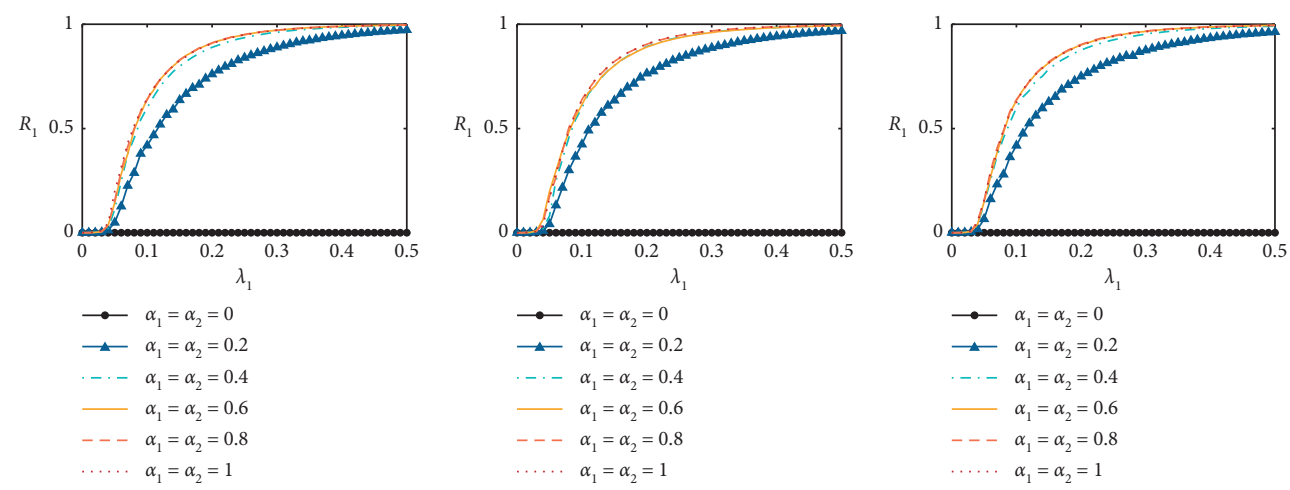

(b)
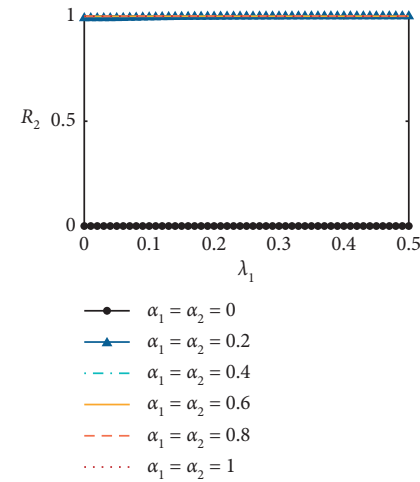

(f)

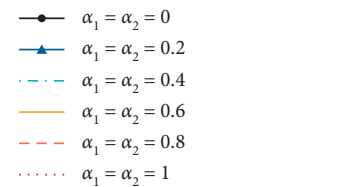

(c)
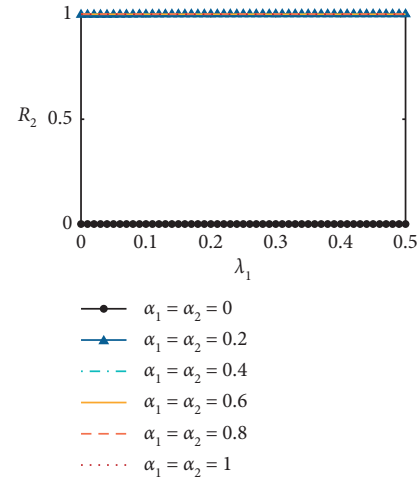

(g)

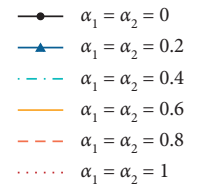

(d)
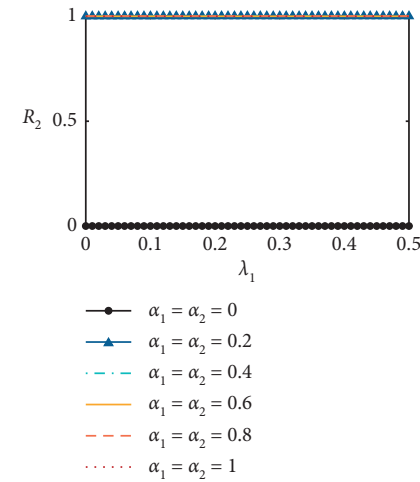

(h)

FIGURE 2: Virus spreading size $R_{1}$ and information spreading size $R_{2}$ versus computer virus transmission probability $\lambda_{1}$ with $q=0.5$. $R_{1}$ versus $\lambda_{1}$ with (a) $\lambda_{2}=0.2$, (b) $\lambda_{2}=0.4$, (c) $\lambda_{2}=0.6$, and (d) $\lambda_{2}=0.8$. $R_{2}$ versus $\lambda_{1}$ with (e) $\lambda_{2}=0.2$, (f) $\lambda_{2}=0.4$, (g) $\lambda_{2}=0.6$, and (h) $\lambda_{2}=0.8$. Other parameters are set to be $\gamma_{1}=\gamma_{2}=0.2$ and $\lambda_{2}=0.8$.

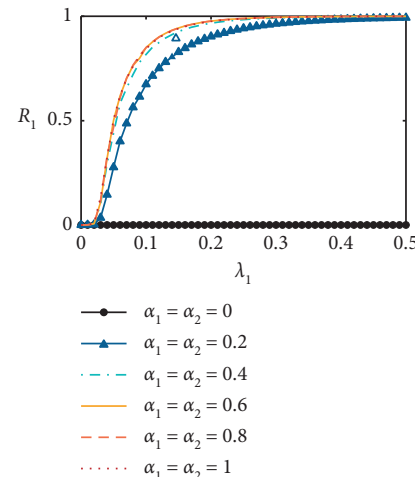

(a)

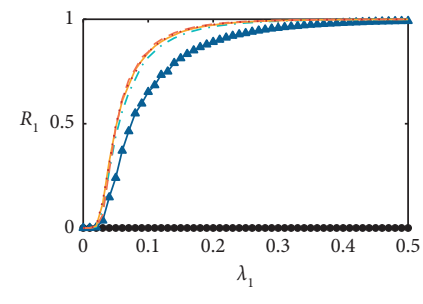

$$
\begin{array}{ll}
\longrightarrow & \alpha_{1}=\alpha_{2}=0 \\
\longrightarrow & \alpha_{1}=\alpha_{2}=0.2 \\
-- & \alpha_{1}=\alpha_{2}=0.4 \\
- & \alpha_{1}=\alpha_{2}=0.6 \\
-- & \alpha_{1}=\alpha_{2}=0.8 \\
\cdots \cdots & \alpha_{1}=\alpha_{2}=1
\end{array}
$$

(b)
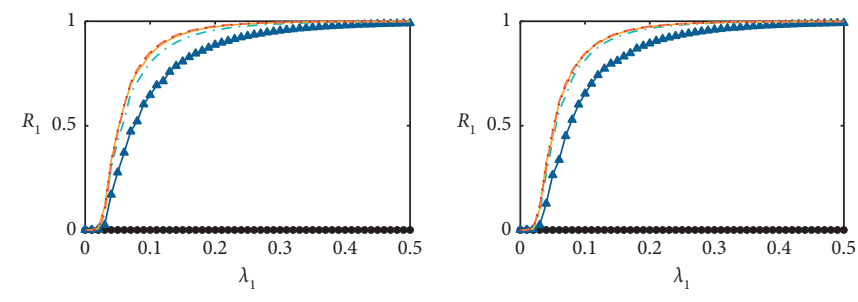

$$
\begin{array}{ll}
\longrightarrow & \alpha_{1}=\alpha_{2}=0 \\
- & \alpha_{1}=\alpha_{2}=0.2 \\
-- & \alpha_{1}=\alpha_{2}=0.4 \\
\longrightarrow & \alpha_{1}=\alpha_{2}=0.6 \\
-- & \alpha_{1}=\alpha_{2}=0.8 \\
\cdots \cdots & \alpha_{1}=\alpha_{2}=1
\end{array}
$$

(c)

$$
\begin{array}{ll}
\longrightarrow & \alpha_{1}=\alpha_{2}=0 \\
\longrightarrow & \alpha_{1}=\alpha_{2}=0.2 \\
-- & \alpha_{1}=\alpha_{2}=0.4 \\
\longrightarrow & \alpha_{1}=\alpha_{2}=0.6 \\
-- & \alpha_{1}=\alpha_{2}=0.8 \\
\cdots \cdots & \alpha_{1}=\alpha_{2}=1
\end{array}
$$

(d)

Figure 3: Continued. 


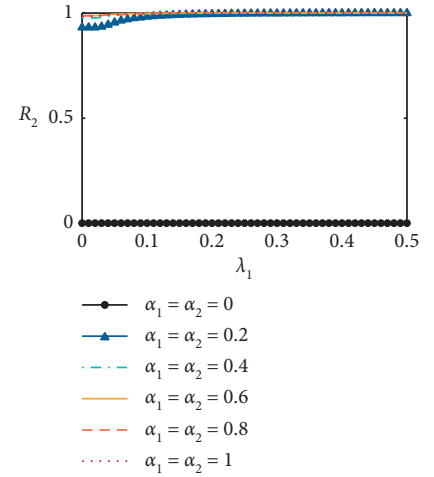

(e)
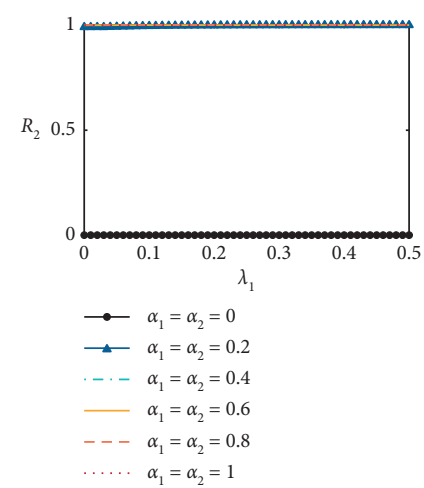

(f)
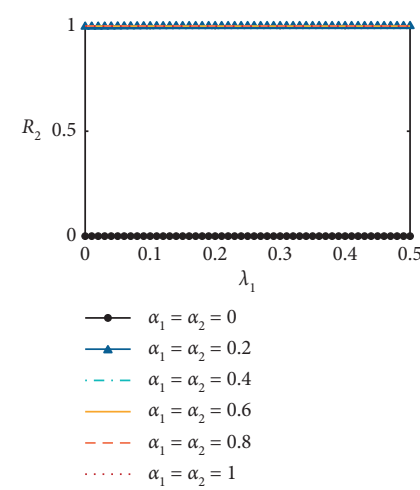

(g)
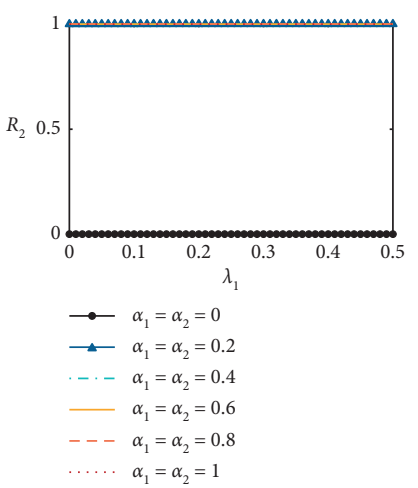

(h)

FIGURE 3: Virus spreading size $R_{1}$ and information spreading size $R_{2}$ versus computer virus transmission probability $\lambda_{1}$ with $q=0.8$. $R_{1}$ versus $\lambda_{1}$ with (a) $\lambda_{2}=0.2$, (b) $\lambda_{2}=0.4$, (c) $\lambda_{2}=0.6$, and (d) $\lambda_{2}=0.8$. $R_{2}$ versus $\lambda_{1}$ with (e) $\lambda_{2}=0.2$, (f) $\lambda_{2}=0.4$, (g) $\lambda_{2}=0.6$, and (h) $\lambda_{2}=0.8$. Other parameters are set to be $\gamma_{1}=\gamma_{2}=0.2$ and $\lambda_{2}=0.8$.

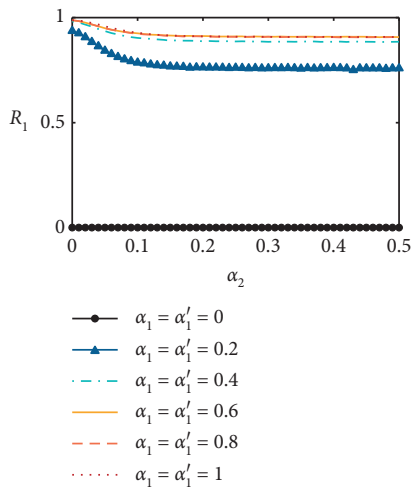

(a)
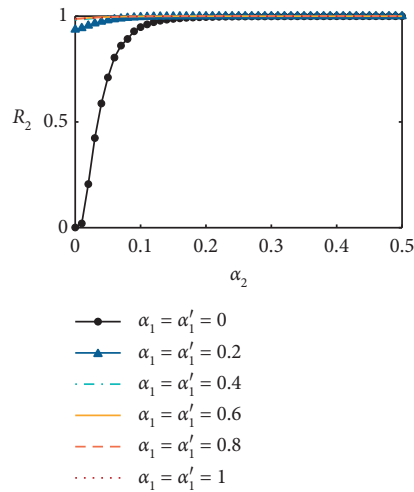

(e)
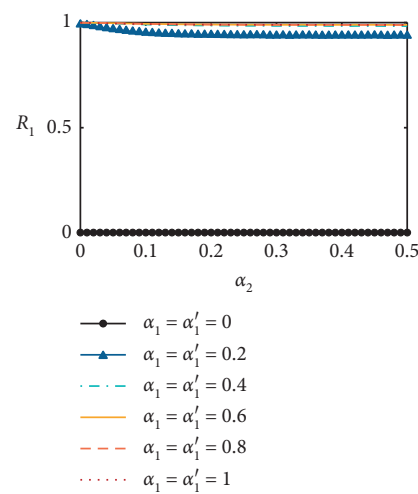

(b)

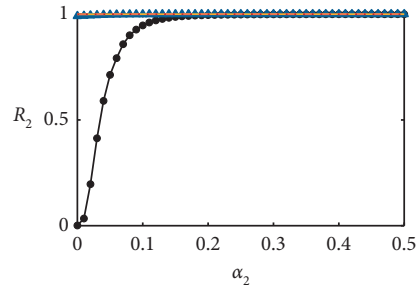

$$
\begin{aligned}
\longrightarrow \alpha_{1}=\alpha_{1}^{\prime}=0 \\
\because-\alpha_{1}=\alpha_{1}^{\prime}=0.2 \\
--\alpha_{1}=\alpha_{1}^{\prime}=0.4 \\
-\alpha_{1}=\alpha_{1}^{\prime}=0.6 \\
--\alpha_{1}=\alpha_{1}^{\prime}=0.8 \\
\ldots \ldots \quad \alpha_{1}=\alpha_{1}^{\prime}=1
\end{aligned}
$$

(f)
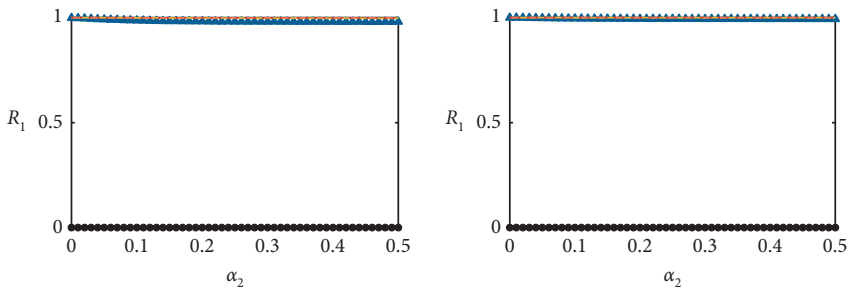

$$
\begin{aligned}
& \longrightarrow \alpha_{1}=\alpha_{1}^{\prime}=0 \\
& \longrightarrow \alpha_{1}=\alpha_{1}^{\prime}=0.2 \\
& \text {-. } \alpha_{1}=\alpha_{1}^{\prime}=0.4 \\
& \text { - } \alpha_{1}=\alpha_{1}^{\prime}=0.6 \\
& \text { - }-\alpha_{1}=\alpha_{1}^{\prime}=0.8 \\
& \alpha_{1}=\alpha_{1}^{\prime}=1
\end{aligned}
$$

(c)
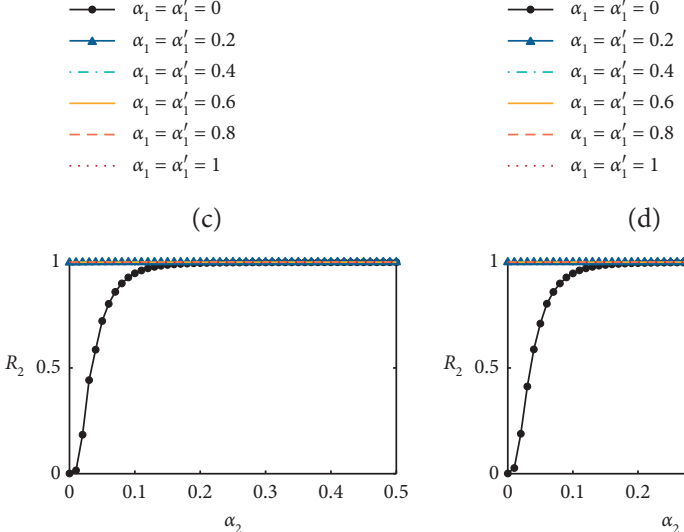

(d)

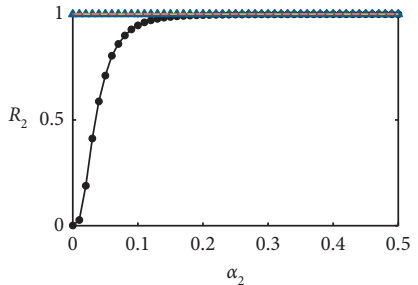

$$
\begin{array}{ll}
\longrightarrow & \alpha_{1}=\alpha_{1}^{\prime}=0 \\
\longrightarrow & \alpha_{1}=\alpha_{1}^{\prime}=0.2 \\
-\cdot- & \alpha_{1}=\alpha_{1}^{\prime}=0.4 \\
- & \alpha_{1}=\alpha_{1}^{\prime}=0.6 \\
-- & \alpha_{1}=\alpha_{1}^{\prime}=0.8 \\
\ldots \cdots & \alpha_{1}=\alpha_{1}^{\prime}=1
\end{array}
$$

(g)

$$
\begin{array}{ll}
\longrightarrow & \alpha_{1}=\alpha_{1}^{\prime}=0 \\
- & \alpha_{1}=\alpha_{1}^{\prime}=0.2 \\
-\cdot- & \alpha_{1}=\alpha_{1}^{\prime}=0.4 \\
- & \alpha_{1}=\alpha_{1}^{\prime}=0.6 \\
-- & \alpha_{1}=\alpha_{1}^{\prime}=0.8 \\
\cdots \cdots & \alpha_{1}=\alpha_{1}^{\prime}=1
\end{array}
$$

(h)

FiguRe 4: Virus spreading size $R_{1}$ and information spreading size $R_{2}$ versus computer virus transmission probability $\lambda_{1}$ with $q=0.5$. $R_{1}$ versus $\alpha_{2}$ with (a) $\lambda_{1}=0.2$, (b) $\lambda_{1}=0.4$, (c) $\lambda_{1}=0.6$, and (d) $\lambda_{1}=0.8$. $R_{2}$ versus $\alpha_{2}$ with (e) $\lambda_{1}=0.2$, (f) $\lambda_{1}=0.4$, (g) $\lambda_{1}=0.6$, and (h) $\lambda_{1}=0.8$. Other parameters are set to be $\gamma_{1}=\gamma_{2}=0.2$ and $\lambda_{2}=0.5$.

$0.8) \geq R_{2}(q=0.5)$ since the promotion of virus spreading on information spreading is decreased.

In Figure 4, we study the effects of susceptibility and infectivity in detail. We find that $R_{1}$ decreases with the increase of susceptibility and infectivity of $L_{2}$. That is to say, the virus spreading can be suppressed by increasing the susceptibility and infectivity. We can explain the results as follows. Increasing susceptibility and infectivity, the information will be widely spread on social network (see Figures $4(\mathrm{e})-4(\mathrm{~g})$ ), and more susceptible nodes in subnetwork $L_{1}$ will take measures to protect themselves from being infected. As a result, $R_{1}$ decreases with $\alpha_{2}$.

Finally, we studied the virus-information spreading as a function of $\alpha_{2}$ when the protection power is lower with $q=0.8$ in Figure 5. We reveal similar phenomena as shown in Figure 4. 


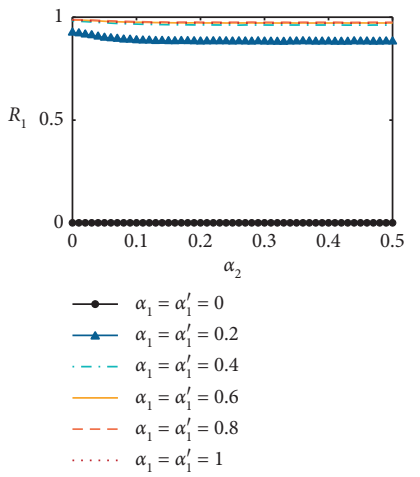

(a)
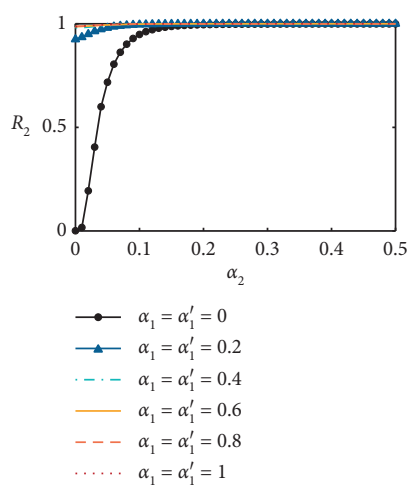

(e)

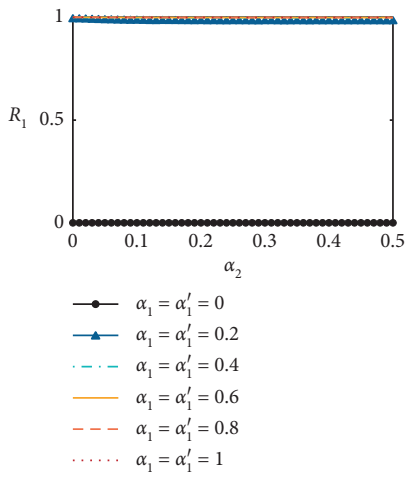

(b)
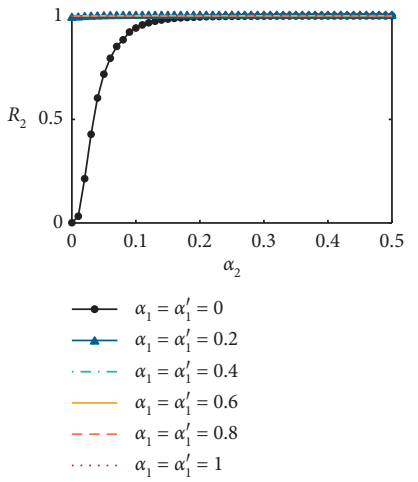

(f)
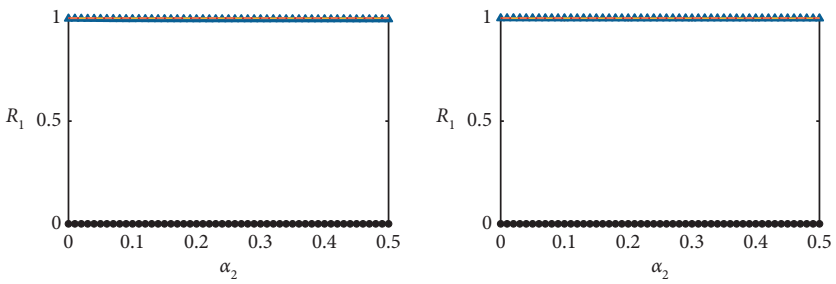

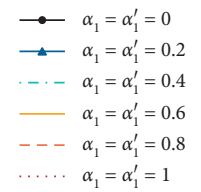

(c)
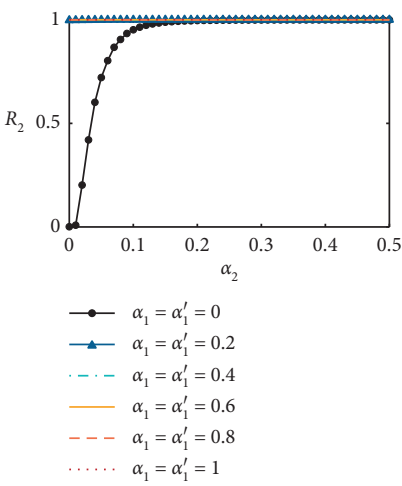

(g)

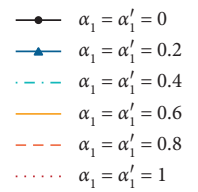

(d)

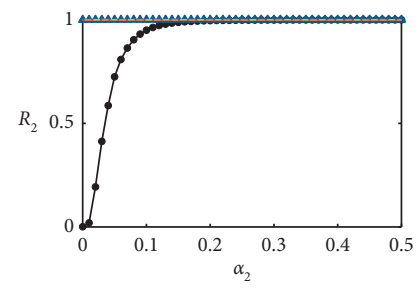

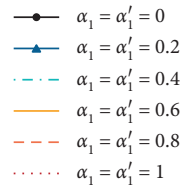

(h)

FIGURE 5: Virus spreading size $R_{1}$ and information spreading size $R_{2}$ versus computer virus transmission probability $\lambda_{1}$ with $q=0.8$. $R_{1}$ versus $\alpha_{2}$ with (a) $\lambda_{1}=0.2$, (b) $\lambda_{1}=0.4$, (c) $\lambda_{1}=0.6$, and (d) $\lambda_{1}=0.8$. $R_{2}$ versus $\alpha_{2}$ with (e) $\lambda_{1}=0.2$, (f) $\lambda_{1}=0.4$, (g) $\lambda_{1}=0.6$, and (h) $\lambda_{1}=0.8$. Other parameters are set to be $\gamma_{1}=\gamma_{2}=0.2$ and $\lambda_{2}=0.5$.

We note that $R_{1}(q=0.5) \geq R_{1}(q=0.8)$ and $R_{2}(q=0.5) \geq R_{2}(q=0.8)$ since the protection power is decreased.

\section{Discussion}

In this paper, we studied the virus-information spreading dynamics on computer-social multiplex networks. We first proposed a mathematical model to describe the coevolution spreading dynamics. In this model, we assumed that nodes' susceptibility and infectivity are heterogeneous and positively correlated with the node's degree. To describe the spreading dynamics, we adopt a generalized heterogeneous mean-field approach. Using extensive numerical simulations, we revealed that the virus spreading dynamics can be significantly suppressed by promoting the information spreading on the computer network or decreasing the susceptibility and infectivity of nodes. Our results provide some insight into containing the virus spreading.

\section{Data Availability}

The data used to support the findings of this study are available from the corresponding author upon request.

\section{Conflicts of Interest}

The authors declare that there are no conflicts of interest regarding the publication of this paper.

\section{Acknowledgments}

This study was partially supported by the Sichuan Science and Technology Program (2020YFG0010).

\section{References}

[1] W. Wang, Q.-H. Liu, J. Liang, Y. Hu, and T. Zhou, "Coevolution spreading in complex networks," Physics Reports, vol. 820, p. 1, 2019.

[2] S. Lehmann and Y.-Y. Ahn, Complex Spreading Phenomena in Social Systems, Springer, Berlin, Germany, 2018.

[3] P. Szor, The Art of Computer Virus Research and Defense: Art Comp Virus Res Defense _p1, Pearson Education, London, UK, 2005.

[4] L.-X. Yang, X. Yang, L. Wen, and J. Liu, "A novel computer virus propagation model and its dynamics," International Journal of Computer Mathematics, vol. 89, no. 17, p. 2307, 2012.

[5] F. D. Sahneh and C. Scoglio, "Competitive epidemic spreading over arbitrary multilayer networks," Physical Review E, vol. 89, Article ID 062817, 2014. 
[6] L.-X. Yang, X. Yang, and Y. Y. Tang, "An effective rumorcontaining strategy," IEEE Transactions on Network Science and Engineering, vol. 5, p. 2, 2017.

[7] C. Granell, S. Gómez, and A. Arenas, "Dynamical interplay between awareness and epidemic spreading in multiplex networks," Physical Review Letters, vol. 111, Article ID 128701, 2013.

[8] C. Granell, S. Gómez, and A. Arenas, "Competing spreading processes on multiplex networks: awareness and epidemics," Physical Review E, vol. 90, Article ID 012808, 2014.

[9] P. C. Ventura, Y. Moreno, and F. A. Rodrigues, "The role of time scale in the spreading of asymmetrically interacting diseases," 2020, http://arxiv.org/abs/2007.02774.

[10] M. E. Newman, "Threshold effects for two pathogens spreading on a network," Physical Review Letters, vol. 95, Article ID 108701, 2005.

[11] M. E. Newman and C. R. Ferrario, "Interacting epidemics and coinfection on contact networks," PLoS One, vol. 8, Article ID e71321, 2013.

[12] B. Karrer and M. E. Newman, "Competing epidemics on complex networks," Physical Review E, vol. 84, Article ID 036106, 2011.

[13] M. Kivelä, A. Arenas, M. Barthelemy, J. P. Gleeson, Y. Moreno, and M. A. Porter, "Multilayer networks," Journal of Complex Networks, vol. 2, no. 3, p. 203, 2014.

[14] M. De Domenico, A. Solé-Ribalta, E. Cozzo et al., "Mathematical formulation of multilayer networks," Physical Review X, vol. 3, Article ID 041022, 2013.

[15] M. De Domenico, V. Nicosia, A. Arenas, and V. Latora, "Structural reducibility of multilayer networks," Nature Communications, vol. 6, p. 1, 2015.

[16] G. Bianconi, Multilayer Networks: Structure and Function, Oxford University Press, Oxford, UK, 2018.

[17] Z. Wang, L. Wang, A. Szolnoki, and M. Perc, "Evolutionary games on multilayer networks: a colloquium," The European Physical Journal B, vol. 88, p. 1, 2015.

[18] X. Zhang, S. Boccaletti, S. Guan, and Z. Liu, "Explosive synchronization in adaptive and multilayer networks," Physical Review Letters, vol. 114, Article ID 038701, 2015.

[19] J. Gao, S. V. Buldyrev, H. E. Stanley, and S. Havlin, "Networks formed from interdependent networks," Nature Physics, vol. 8, no. 1, p. 40, 2012.

[20] D. Zhou, J. Gao, H. E. Stanley, and S. Havlin, "Percolation of partially interdependent scale-free networks," Physical Review E, vol. 87, Article ID 052812, 2013.

[21] F. Radicchi and A. Arenas, "Abrupt transition in the structural formation of interconnected networks," Nature Physics, vol. 9, no. 11, p. 717, 2013.

[22] S. Gomez, A. Diaz-Guilera, J. Gomez-Gardenes, C. J. PerezVicente, Y. Moreno, and A. Arenas, "Diffusion dynamics on multiplex networks," Physical Review Letters, vol. 110, Article ID 028701, 2013.

[23] M. Dickison, S. Havlin, and H. E. Stanley, "Epidemics on interconnected networks," Physical Review E, vol. 85, Article ID 066109, 2012.

[24] M. Pósfai, J. Gao, S. P. Cornelius, A.-L. Barabási, and R. M. D’Souza, "Controllability of multiplex, multi-timescale networks," Physical Review E, vol. 94, Article ID 032316, 2016.

[25] L. V. Gambuzza, M. Frasca, and J. Gómez-Gardeñes, "Intralayer synchronization in multiplex networks," EPL (Europhysics Letters), vol. 110, no. 2, Article ID 20010, 2015.

[26] L. Tang, X. Wu, J. Lü, J.-a. Lu, and R. M. D’Souza, “Master stability functions for complete, intralayer," Physical Review E, vol. 99, Article ID 012304, 2019.
[27] S. Jalan, V. Rathore, A. D. Kachhvah, and A. Yadav, "Inhibition-induced explosive synchronization in multiplex networks," Physical Review E, vol. 99, Article ID 062305, 2019.

[28] Z. Wang, Q. Guo, S. Sun, and C. Xia, "The impact of awareness diffusion on SIR-like epidemics in multiplex networks," Applied Mathematics and Computation, vol. 349, p. 134, 2019.

[29] C. Zheng, C. Xia, Q. Guo, and M. Dehmer, "Interplay between SIR-based disease spreading and awareness diffusion on multiplex networks," Journal of Parallel and Distributed Computing, vol. 115, p. 20, 2018.

[30] J.-Q. Kan and H.-F. Zhang, "Effects of awareness diffusion and self-initiated awareness behavior on epidemic spreading-an approach based on multiplex networks," Communications in Nonlinear Science and Numerical Simulation, vol. 44, p. 193, 2017.

[31] E. Massaro and F. Bagnoli, "Epidemic spreading and risk perception in multiplex," Physical Review E, vol. 90, Article ID 052817, 2014.

[32] R. Pastor-Satorras and A. Vespignani, "Epidemic dynamics and endemic states in complex networks," Physical Review E, vol. 63, Article ID 066117, 2001.

[33] R. Pastor-Satorras and A. Vespignani, "Epidemic dynamics in finite size scale-free networks," Physical Review E, vol. 65, Article ID 035108, 2002.

[34] R. Pastor-Satorras, C. Castellano, P. Van Mieghem, and A. Vespignani, "Epidemic processes in complex networks," Reviews of Modern Physics, vol. 87, no. 3, p. 925, 2015.

[35] J. C. Miller, "Natural visions: the power of images in american environmental reform," Physical Review E, vol. 76, Article ID 010101, 2007.

[36] M. Catanzaro, M. Boguñá, and R. Pastor-Satorras, "Diffusionannihilation processes in complex networks," Physical Review E, vol. 71, Article ID 027103, 2005.

[37] Z.-K. Zhang, C. Liu, X.-X. Zhan, X. Lu, C.-X. Zhang, and Y.-C. Zhang, "Dynamics of information diffusion and its applications on complex networks," Physics Reports, vol. 651, p. 1, 2016.

[38] W. Wang, M. Tang, H. E. Stanley, and L. A. Braunstein, "Unification of theoretical approaches for epidemic spreading on complex networks," Reports on Progress in Physics, vol. 80, Article ID 036603, 2017.

[39] Y. Moreno, R. Pastor-Satorras, and A. Vespignani, "Epidemic outbreaks in complex heterogeneous networks," The European Physical Journal B, vol. 26, no. 4, p. 521, 2002.

[40] B. Karrer and M. E. J. Newman, "Random graphs containing arbitrary distributions of subgraphs," Physical Review E, vol. 82, Article ID 016101, 2010. 